DOI: $10.2478 / \mathrm{v} 10282-012-0007-0$

\title{
Meningioma of the pineal region
}

\author{
M. Radoi, L. Danaila, F. Stefanescu, R. Vakilnejad, Roxana Popa \\ Neurosurgery Clinic of the National Institute of Neurology and Neurovascular \\ Diseases, Bucharest
}

\begin{abstract}
Meningiomas rarely occur in the pineal region, but they can reach huge diameters. We presented the case of a patient with a very large meningioma of the pineal region $(6 \times 5 \times 4 \mathrm{~cm})$. The tumor, developed from the falcotentorial junction, was totally removed via an occipital interhemispheric transtentorial approach with minimal postoperative neurological deficits. The postoperative course was complicated with an acute internal hydrocephalus that needed temporary placement of an external ventricular drainage. The supratentorial surgical corridors allow for increased exposure and are best suited for falcotentorial meningiomas.
\end{abstract}

\section{Introduction}

Pineal region tumors are very rare comprising about $1 \%$ of all intracranial neoplasms (10). Neoplasms of the pineal region are of a great hystological variety, which suggest their origin either from the pineal parenchymals cells or from other cell lines (astrocytes, germ cells, arachnoid cells). Among this variety of tumors, meningiomas of the third ventricle and pineal gland have been only rarely reported in the literature

Cushing and Eisenhardt (7), in an extensive series of meningiomas, showed no cases of pineal region meningiomas. Sachs et al. (21) reported three cases of pineal region meningiomas in 1962 and found only 20 cases in the literature at that time. In a similar review, Piatt and Campbell (19) revealed in 1983, 37 cases from the literature, including two of their own. In the recent neurosurgical literature, meningiomas of the pineal region are, usually, presented as case reports, included $1-3$ cases per report $(4,13,24)$.

In this report, we presented our surgical experience with one case of a very large pineal meningioma.

\section{Case report}

We reported the case of a 67 years old male patient, with a one year history of headache and deficits in memory, symptoms that worsen progressively, being associated in the last month with diplopia and spontaneous tremor in the right hand.

On admission in our clinic, the neurological examination revealed irregular balance disorders, without motor deficits, bilateral cutaneous plantar reflex in extension, horizontal nystagmus and vertical paresis of vision (Parinaud syndrome). Visual acuity decreased in the right eye, but the visual field was normal. Fundus examination revealed bilateral papillary oedema. As his psychic was concerned, the patient was alert and oriented (GCS $=15$ pct.).

Cerebral CT-scan examination showed a well circumscribed tumor, which measured $6 \times 5 \times 4 \mathrm{~cm}$. in diameters, located 
periventricular in the pineal region, with uniform and intense contrast enhancement (Figure 1 A, B). The tumor presented a small, central, hyperdense area (calcification) and produced moderate supratentorial obstructive hydrocephalus. Cerebral angiography showed that the internal cerebral veins and vein of Galen were stretched and significantly displaced downwords.

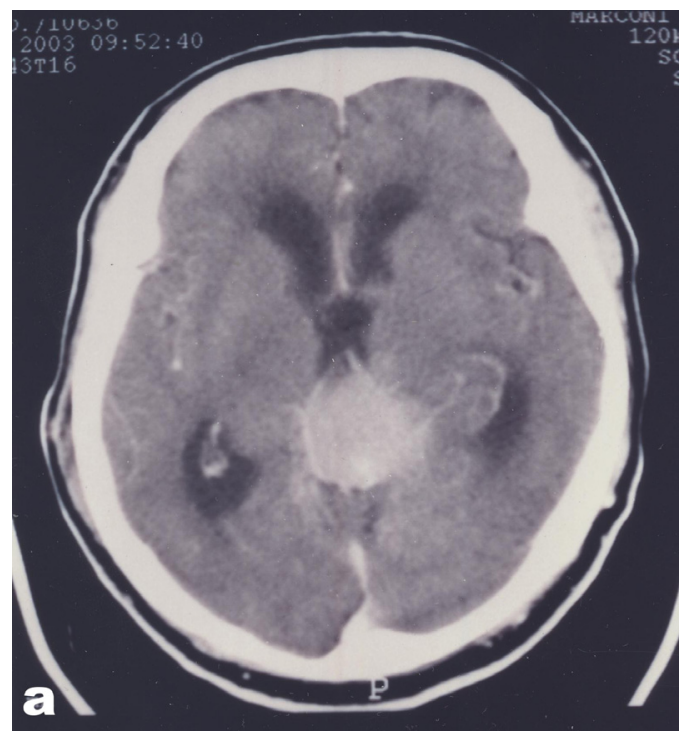

A

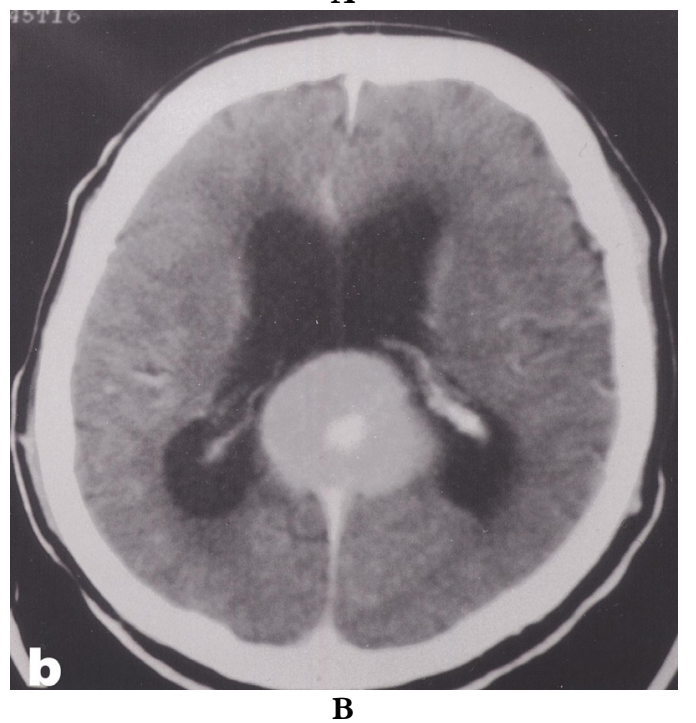

Figures 1 A, B Axial contrast cerebral CT-scan showing a well circumscribed, approximately $6 \mathrm{~cm}$. in diameter meningioma, located periventricular in the pineal region, with uniform and intense contrast enhancement

The patient was operated using a right occipital interhemispheric transtentorial approach. The tumor was a firm, vascularized tissue mass, whitish-yellowish in colour. Under microscope magnification we performed a total resection of the tumor. The histological exam revealed a fibrous meningioma with colagenic proliferation areas.

After a good recovery in the first postoperative day, without neurological deficits, the patient became somnolent and soon he lapsed into a superficial coma state. Thus, 48 hours after surgery, we performed a cerebral CT scan, which showed an acute internal hydrocephaly. An external ventricular drainage was performed in emergency. In the following days the neurological recovery of the patient was good. The external ventricular drainage was maintained 6 days, and after its removal a cerebral CT-scan was performed, which showed the reduction of the ventricular size.

When he had left the hospital, twenty days after the first operation, the patient presented only a moderate left hemiparesis and left homonymus hemianopsia. Periodical postoperative follow-ups, at 6, 12 and respectively 24 months demonstrated a good general and neurological condition of the patient with minor neurological deficits. Control cerebral CT-scans showed moderate internal hydrocephalus, without signs of periventricular cerebrospinal fluid resorbtion, and absence of any local recurrence (Figure $2 \mathrm{~A}, \mathrm{~B}$ ). 
DOI: $10.2478 / v 10282-012-0007-0$

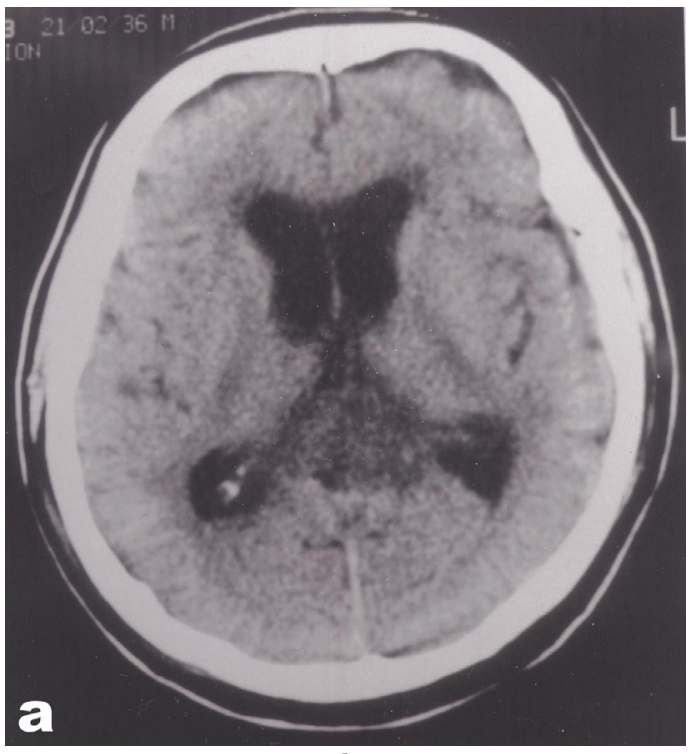

A

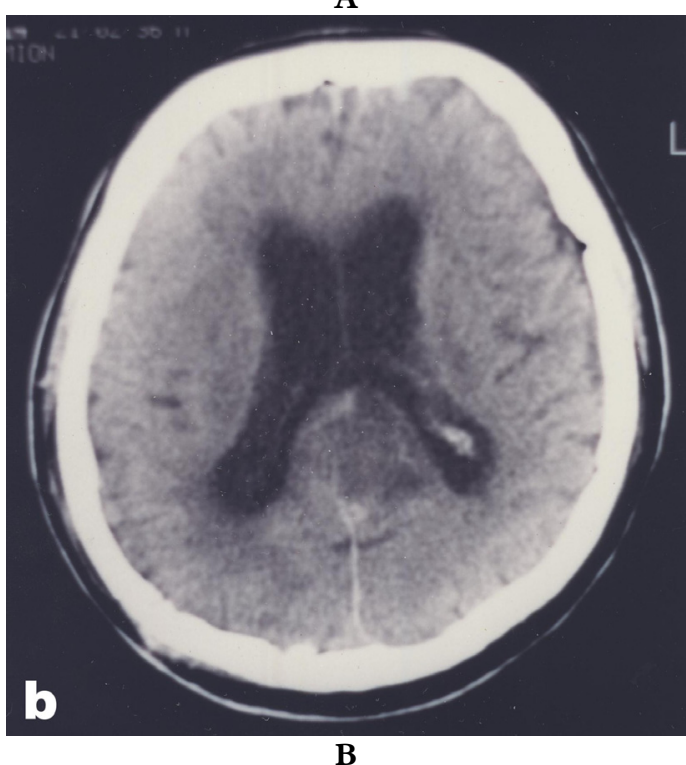

Figures 2 A, B Axial contrast cerebral CT-scan, performed 20 months after the operation, showed reduced hydrocephaly and absence of any intracerebral tumoral mass in the pineal region

\section{Discussion}

Meningiomas of the pineal region could be divided into two major groups, depending on their origin:
1. meningiomas arising from the free edge of the tentorium, where it is joined by the inferior margin of the falx (falcotentorial jonction); they secondarily invade the pineal and posterior third ventricular region and there were authors who considered that pineal region meningiomas are, in fact, extensions of meningiomas of the posterior falx or midline tentorial meningiomas $(6,17)$

2. meningiomas developed from the velum interpositum, the space between the two layers of the tela choroidea. These meningiomas have no dural attachment because tela choroidea is formed only by the pial and arachnoidal layer of the meninges; they are exclusively located in the posterior third ventricle, presenting as a pineal region mass and should be distinguished from those developing in the anterior third ventricle (6).

An important difference between the categories that comprise meningiomas of the pineal region, despite their dural attachments, lies in the origin of their blood supply. The velum interpositum meningioma, attached to the tela choroidea, receives its blood supply from the posterior choroidal arteries and, if the tumor extends anteriorly, from the anterior choroidal artery. Meningiomas arising from the free edge of the tentorium derive their blood supply from branches of the internal carotid artery supplying the falx and tentorium, of which the Bernasconi and Cassinari artery is the most important (18).

Patients with meningioma are usually older than those with other pineal region tumors; there is also a female predominance (14). The most frequent clinical symptoms are related to raise intracranial pressure from insidious hydrocephalus. These most often include headache, gait disturbance, cognitive dysfunction and papilledema. Other onset symptoms include signs of 
ataxia, visual disturbances, hearing loss, hemifacial spasm or vertical paresis of vision (Parinaud's syndrome) (1).

In pineal region meningiomas, the cytological examination of the cerebrospinal fluid and the dosage of the malignant germcell markers ( $\alpha$-fetoprotein and $\beta$-human chorionic gonadotropin) in the patients' blood are negative and provide little diagnostic value in limiting the differential diagnosis.

\section{Therapeutic considerations}

The location of the pineal region in the centre of the brain, above the brain stem, presents a surgical challenge to safely expose and resect a pineal region tumor. The goal of surgery for pineal region tumor vary depending on the clinical circumstances of an individual situation. Thus, the prognosis for pineal meningiomas became excellent. Many are encapsulated, affording a direct microsurgical exposure and disection, and a gross total resection.

A minimum goal should be to establish a histologic diagnosis by stereotactic biopsy. It provides direct tumoral tissue sampling for diagnosis and guides further operative or nonoperative therapy (3). Al-Mefty and al. sustain that if neuroradiologic image is pathognomonic for a meningioma, it is advisable to proceed to direct microsurgical approaches (2).

Complete resection of pineal region meningiomas depends upon adherence of the tumoral mass to the surrounding vital structures. In general, gross total resection of velum interpositum meningiomas is more likely than of the falcotentorial variety, in which case dural tentorial adherence and venous sinus involvment create the potential for subtotal resection.

Surgical approaches to the pineal region include the supratentorial and infratentorial approaches. The infratentorial supracerebellar approach is well suited for meningiomas arising from velum interpositum, without dural attachment. The supratentorial approaches are more complicated because of their variable anatomy and are best suited for falcotentorial meningiomas, as they allow intraoperative options for tentorial or falx incisions and permit the deprivations of the tumor's blood supply at its base.

Internal hydrocephalus occurs frequently in patients with neoplasm of the pineal region. The choice of optimal surgical attitude varies from case to case. In patients having minimal neurological signs or when a strong suspicion of a potentially benign, excisable tumor exists, direct surgery is the first option, because the removal of the tumor relive hydrocephalus or a temporary ventricular drain can be placed at the time of surgical resection. In patients who have signs of an acute hydrocephalus and in whom a reduction in intracranial pressure is desirable prior to an open resection, a CT- guided stereotactic endoscopic third ventriculostomy is the method of choice (11).

The choice of optimal approach depends on the anatomical extent of the meningioma, its relation with the surrounding structures, its presumed origin (falcotentorial or velum interpositum) as well as the preference and experience of the operating neurosurgeon $(9,20)$. In our case we chose a supratentorial occipital linterhemispheric transtentorial approach because we considered that the meningioma developed from the falcotentorial margin. In a brief description we presented some advantages and disadvantages of the main operative approaches.

A) The infratentorial supracerebellar approach to the pineal region was first utilized by Krause in the 1920s and 
DOI: $10.2478 / \mathrm{v} 10282-012-0007-0$

developed and popularized by Stein in the 1970 s $(26,27)$. It is indicated for small meningiomas arising from the velum interpositum. This approach presents the following advantages: (1) - a direct central midline approach to the tumor, (2) avoidance of injury to the deep venous system since the tumors are ventral to the deep venous system, (3) - has no morbidity related to the parietal or occipital lobe retraction. As disadvantages of this approach could be mentioned: (1) - the narrow space to expose the meningioma, because of the small distance between the coliculi and tentorial hiatus, (2) - the sacrifice of vermian veins, (3) - the difficulty to reach lesions above the tentorial notch.

B) The supratentorial approach to the pineal region was first performed by Dandy (11) in 1921, then by Kunicki (18) and others. Dandy utilized a large parietooccipital bone flap and a parasagittal approach to the pineal region. After opening the dura, multiple opperative corridors may be developed depending on the size and location of the tumor in order to allow an optimal intraoperative exposure of the pineal region. These include a tentorial incision, a falcine incision, splenial retraction or incision of the posterior body of the corpus callosum $(4,8,14,24)$ :

a) The occipital transtentorial approach is indicated for meningiomas arising primarily from the free edge of the tentorium. This approach provides an excellent view both above and below the tentorial notch, a great exposure of the lesion and view of the quadrigeminal region. Choosing this approach may damage the occipital lobe and the splenium of the corpus callosum and, also, it may be difficult to reach parts of the meningioma extending to the opposite side. b) The transcallosal interhemispheric approach is the most suitable for large meningiomas, located in the pineal region, in the posterior third ventricle or tumors extending anterior to the level of the adhesio interthalamica. This approach has the advantage of providing an excellent view of meningiomas located in the posterior third ventricle. The main disadvantage is the possible damage of the posterior portion of the corpus callosum or the fornix.

The occipital transtentorial approach can be performed with the patient in sitting or the prone position. Aussman (4) described a three-quarter prone position for the occipital transtentorial approach to eliminate the risk of embolism. Despite the fact the prone position eliminates the need for retraction of the occipital lobe due to gravity and is more comfortable for the surgeon, Konvalov (14) prefers the occipital transtentorial approach in sitting position, because the visualization of the controlateral Rosenthal vein and surgeon intraoperative orientation are more difficult in prone/three-quarter prone positions. We chose the prone position for our occipital transtentorial approach.

Sekhar (24) and Ziyal et al (29) described transsinus transtentorial approaches to large pineal meningiomas in 1992. After removal of the tumor they resutured the transverse sinus to reestablish the blood circulation. Kawashima et al (13) proposed a new approach, but only at the level of anatomical study, called the occipital bitranstentorial falcine approach, which is a modification of occipital transtentorial approach.

In the same way, in 1990, Van den Berg proposed the lateral-paramedian infratentorial approach, which is a modification of the infratentorial supracerebellar approach (28). Thus, the 
unilateral cerebellopontine angle is explored, and from there, the tentorial notch is reached over the ipsilateral cerebellar hemisphere. The meningioma can be dissected between the internal cerebral vein and the basal vein of Rosenthal.

Adjuvant therapy in the treatment of pineal meningiomas has enhanced the possibility of cure or, at least, has improved the postoperative clinical results. For subtotal resected meningiomas radiation therapy should be utilized, either by conventional means or stereotactic radiosurgery. Several retrospective studies have supported the use of radiotherapy in the management of pineal meningiomas following subtotal resection (5). This result also applied to subtotal resection following surgery for recurrent meningioma. Radiotherapy is indicated for the overall management of aggressive meningiomas (22).

Taking into account the difficulties of a direct surgical approach to the pineal region, for meningiomas less than $2-3 \mathrm{~cm}$. in size or for the recurrent ones, stereotactic surgery could be a valid solution (5).

\section{Prognosis}

The neoplasms of the pineal region have an overall operative mortality in the range of $3 \%$, with morbidity in the range of $4 \%$ (27). Patients with pineal region tumors have a five-year survival in the range of 55\% (1). A favourable outcome occurs over $90 \%$ of the time (23). Among these, meningiomas, especially total removed meningiomas, have a very good long-term prognosis. In case of pineal region meningiomas, benign and encapsulated tumors, the success rate approaches 100\% $(4,13)$.

The long-term prognosis and risk of recurrence was directly related to the extent of surgical resection and the completeness of resection (25). Atypical or anaplasic meningiomas have an increased risk of recurrence $(12,27)$. Significant prognostic factors for a pineal region meningioma are: (1) - the extent of removal, (2) preoperative clinical condition, (3) postoperative clinical condition and (4) histological type.

\section{Conclusions}

Pineal region surgery, in which the experience of the neurosurgical team plays a very important role, was continually successful in the last years. Total or partial resection of pineal meningioma by direct neurosurgical approaches can be achieved in most cases with low morbidity and depend upon the tumor's dural attachment at its base and its relationship with the surrounding vital structures. Usually, the total removal of the pineal region meningioma reestablish the cerebrospinal circulation at the end of the operation.

Large pineal region meningioma (> $5 \mathrm{~cm}$ ) should be operated through a unilateral or bilateral occipital interhemispheric transtentorial approach, regardless of the angiographic findings, because this provide a wide operative field and can be followed when necessary, by an infratentorial supracerebellar approach.

Corresponding author: Dr. Radoi Mugurel

E-mail address-muguradoi@yahoo.com

\section{References}

1. Allen JC, Bruce JN, Kun LE, Langford LA. Pineal region tumors. In : Levin VA, ed. Cancer in the nervous system, New York: Churchill Livingstone, 1996: 171186.

2. Al-Mefty et al. Meningiomas. In: Raven Press, Ltd., New York, 1991: 583-591.

3. Apuzzo MLJ, Chandrasoma PT, Breeze RE, Cohen DM, Luxton G, Mazumder A. Applications of imagedirected stereotactic surgery in the management of intracranial neoplasms. In: Heilbrun PM (ed). Concepts in neurosurgery: stereotactic neurosurgery, vol 2 . Baltimore: Williams\&Wilkins, 1988; 73-132. 
DOI: $10.2478 / v 10282-012-0007-0$

4. Ausman JI, Malik JM, Dujovny M, Mann R. Pineal region: rare location of a cavernous haemangioma. Neurol Res 16 (2); 133-136, 1994.

5. Bararo NM, Gutin PH, Wilson CB, Sheline JE, Boldrey EB, Wara WM. Radiation therapy in the treatment of partially resected meningiomas. Neurosurgery 1987; 20: 525-528.

6. Camins MB, Schlesinger EB. Treatment of tumors of the posterior part of the third ventricle and the pineal region: a long-term follow-up. Acta Neurochir (Vienna) 1978; 40: 131-143.

7. Cushing H, Eisenhardt L. Meningiomas: their classification, regional behaviour, life history, and surgical end results. Springfield, Illinois: Charles C. Thomas, 1938

8. Danaila L, Petrescu DA, Stefanescu F. Our experience in surgery of tumors of the third ventricle. Rom Neurosurg 1997; new series 1-2: 49-61.

9. Dandy WE. An operation for the removal of pineal tumors. Surg Gynecol Obstet 1921; 33: 113-119.

10.Edwards MSB, Hudgins RJ, Wilson CB, Levin VA,

Wara WM. Pineal region tumors in children. J Neurosurg 1988; 68: 689-697.

11.Goodman R. Magnetic resonance imaging-directed stereotactic endoscopic third ventriculostomy. Neurosurgery, 1993; 32: 1043-1047.

12.Jaaskelainen J, Haltia M, Servo A. Atypical and anaplastic meningiomas: radiology, surgery, radiotherapy and outcome. Surg Neurol 1986; 25: 233 242.

13.Kawashima M, Rhoton AL Jr, Matsushima $\mathrm{T}$ : Comparison of posterior approaches to the posterior incisural space: microsurgical anatomy and proposal of a new method, the occipital bi-transtentorial/falcine approach. Neurosurgery 51 (5); 1208-1220, 2002.

14.Konovalov AN, Spallone A, Pitzkhelauri DI: Meningioma of the pineal region:a surgical series of 10 cases. J Neurosurg 85;586-590, 1996.

15.Kunicki A: Operative experience in 8 cases of pineal tumors. J Neurosurg 17:815-823, 1960.

16.Markwalder TM, Markwalder RV, Markwalder HM.
Meningioma of the anterior part of the third ventricle: case report. J Neurosurg 1979; 50: 233-235.

17.Obrador S, Soto M, Gutierrez-Diaz JA. Surgical management of tumours of the pineal region. Acta Neurochir (Vienna) 1976; 34: 159-171

18.Papo I, Salvolini U. Meningiomas of the free margin of the tentorium developing in the pineal region. Neuroradiology 1974; 7: 237-243.

19.Piatt JH Jr, Campbell GA. Pineal region meningioma: report of two cases and literature review. Neurosurgery 1983; 12: 369-376.

20.Rhoton AL Jr. Microsurgical anatomy of the third ventricular region. In: Apuzzo MLJ (ed). Surgery of the third ventricle. Baltimore: Williams\&Wilkins, 1987: 92166.

21.Sachs E Jr, Avman N, Fischer RG. Meningiomas of the pineal region and posterior part of 3rd ventricle. J Neurosurg 1962; 19:325-331.

22.Salazar OM. Ensuring local control in meningiomas. Int J Radiat Oncol Biol Phys 1988; 15: 501-504.

23.Schild SE, Scheithauer BW, Schomberg PJ, et. al. Pineal parenchimal tumors: clinical, pathologic, and therapeutic aspects. Cancer 1993; 72: 870-880.

24.Sekhar LN, Goel A. Combined supratentorial and infratentorial approach to large pineal meningioma . Surg Neurol 37 (3); 197-201, 1992.

25.Simpson D. The recurrence of intracranial meningiomas after surgical treatment. J Neurol Neuropsychiatry 1957; 20: 22-39.

26.Stein BM. Infratentorial supracerebellar approach. In: Apuzzo MLJ (ed). Surgery of the third ventricle. Baltimore: Williams\&Wilkins, 1987; 570-590.

27.Stein BM, Bruce JN. Surgical management of pineal region tumors. In: Clinical neurosurgery, vol 39, Williams\&Wilkins, 1992: 509-532.

28.Van der Bergh R. Lateral-paramedian infratentorial approachin lateral decubitus for pineal tumors. Clinical Neurology and Neurosurgery 92 (4); 311-316, 1990.

29.Ziyal IM, Sekhar LN, Salas E, Olan WJ. Combined supra/infratentoria-transsinus approach to large pineal region tumors. J Neurosurg 88; 1050-1057, 1998. 